\title{
Editorial: Redox Homeostasis Managers in Plants under Environmental Stresses
}

\author{
Naser A. Anjum ${ }^{1 *}$, Nafees A. Khan ${ }^{2 *}$, Adriano Sofo ${ }^{3 *}$, Margarete Baier ${ }^{4 *}$ and Rene Kizek ${ }^{5 *}$ \\ ${ }^{1}$ Centre for Environmental and Marine Studies and Department of Chemistry, University of Aveiro, Aveiro, Portugal, \\ ${ }^{2}$ Department of Botany, Aligarh Muslim University, Aligarh, India, ${ }^{3}$ School of Agricultural, Forestry, Food and Environmental \\ Sciences, University of Basilicata, Potenza, Italy, ${ }^{4}$ Plant Physiology, Dahlem Centre of Plant Sciences, Institute of Biology, \\ Freie Universität Berlin, Berlin, Germany, ${ }^{5}$ Department of Human Toxicology and Pharmacology, Faculty of Pharmacy, \\ University of Veterinary and Pharmaceutical Sciences, Brno, Czech Republic
}

Keywords: redox homeostasis, plant life, oxidative stress, environmental stress, redox couples

OPEN ACCESS

Edited by:

Bruno Silva Nunes,

University of Porto, Portugal

Reviewed by:

Miguel Machado Santos,

University of Porto, Portugal

Glória Catarina Pinto,

University of Aveiro, Portugal

*Correspondence:

Naser A. Anjum anjum@ua.pt;

Nafees A. Khan

naf9@lycos.com;

Adriano Sofo

adriano.sofo@unibas.it;

Margarete Baier

margarete.baier@fu-berlin.de;

Rene Kizek

kizek@sci.muni.cz

Specialty section:

This article was submitted to

Environmental Toxicology,

a section of the journal

Frontiers in Environmental Science

Received: 18 March 2016

Accepted: 28 April 2016

Published: 13 May 2016

Citation:

Anjum NA, Khan NA, Sofo A, Baier M and Kizek $R$ (2016) Editorial: Redox

Homeostasis Managers in Plants under Environmental Stresses.

Front. Environ. Sci. 4:35. doi: 10.3389/fenvs.2016.00035

\section{The Editorial on the Research Topic}

\section{Redox Homeostasis Managers in Plants under Environmental Stresses}

Environmental stresses, grouped broadly into abiotic (physical environment e.g., drought, temperature regimes, UV-B radiation, salinity, and metals/metalloids) and biotic (e.g., pathogen, herbivore) significantly modulate the survival, reproduction, and productivity of plants/crops (Redondo-Gómez, 2013). In particular, environmental stresses caused by climate change, such as drought, high salinity, and low and high temperatures are predicted to become more severe and widespread (Osakabe et al., 2013). At cellular level, the sustenance of plant life under stressful environment is controlled by homeostasis in the usual redox reactions. Redox reactions can contribute various reactive oxygen species (ROS). One-, two-, and three-electron reduction of $\mathrm{O}_{2}$ or excitation of triplet oxygen $\left({ }^{3} \mathrm{O}_{2}\right)$ can occur and cause the formation of superoxide radical $\left(\mathrm{O}_{2}^{\bullet-}\right)$ or hydroperoxyl radical $\left(\mathrm{HO}_{2}^{\bullet}\right)$, hydrogen peroxide $\left(\mathrm{H}_{2} \mathrm{O}_{2}\right)$, hydroxyl radical $\left(\bullet^{\bullet} \mathrm{OH}\right)$, and singlet oxygen $\left({ }^{1} \mathrm{O}_{2}\right)$, respectively. As integral signaling molecules, ROS regulate growth and development of plants, and also modulate their responses to biotic and/or abiotic stimuli (Baxter et al., 2014). However, beyond their steady-state cellular concentrations, ROS and their reaction products can modulate plant stress responses and/or severely impair the cellular redox homeostasis (Dietz, 2003; Oelze et al., 2008; Baxter et al., 2014; Juszczak et al.). Nevertheless, extreme environmental conditions are inevitable for plants and can excessively over-reduce or overoxidize cellular environment. Previous conditions are likely to cause an imbalance in the generation and metabolism of ROS (and their reaction products), loss in the cellular redox homeostasis and finally the arrest in the cellular metabolism (Foyer and Noctor, 2009, 2012).

Plants are equipped with several strategies to efficiently metabolize and tightly regulate the steady-state levels of cellular ROS (and its reaction products), and manage cellular redox homeostasis at its optimum. The list of major cellular redox homeostasis managers includes redoxrelated components such as proteinaceous thiol members such as thioredoxin, glutaredoxin, and peroxiredoxin proteins, and key soluble redox-compounds such as ascorbate (AsA) and glutathione (GSH). Electron carriers and energy metabolism mediators such as non-phosphorylated (NAD+) and the phosphorylated (NADP+) coenzyme forms and their redox couples DHA/AsA, GSSG/GSH, NAD+/NADH, and NADP+/NADPH also contribute to cellular redox homeostasis (Schafer and Buettner, 2001; Anjum et al., 2010, 2012a,b; Foyer and Noctor, 2011, 2012; Suzuki et al., 2012; Giordano, 2013). The cellular redox homeostasis has also been regarded as the major "integrator" of information from metabolism and the plant-environment relationship 
(Foyer and Noctor, 2009). Hence, unveiling insights into the role and underlying mechanisms of redox homeostasis in plants under environmental stresses has been the major focus of current plant research (Schafer and Buettner, 2001; Foyer and Noctor, 2009, 2011, 2012; Anjum et al., 2010, 2012a,b; Suzuki et al., 2012; Giordano, 2013).

The present volume "Redox Homeostasis Managers in Plants under Environmental Stresses" updates the readers on the subject, and discusses research progress and current understanding on the subject. Among the significant 17 article types, original research reports exemplified new information on how redox homeostasis managers are modulated by environmental cues and what potential strategies are useful for improving cellular concentrations of major redox homeostasis managers. Additionally, detailed updates to specific topics and so far unexplored aspects were highlighted by focused review/mini-review articles.

Cellular redox homeostasis is impacted by abiotic factors that can cause elevations in ROS (and their reaction products) at varying levels in the major energy organelles, such as chloroplast and mitochondria (Das et al.). Soil salinity is a serious threat to crop productivity worldwide that causes oxidative stress through imposing ion toxicity, osmotic stress, and metabolic imbalance (Adem et al., 2014; Abd Elgawad et al.). Notably, salinity stress significantly impacts electron flow in the electron transport chain in these organelles, disturbs the status of adenine (ATP) and pyridine nucleotides (NADH, NADPH), and eventually leads to elevation in cellular ROS and lipid peroxidation (LPO; Srivastava et al.). However, the extent of salinity-impact on ATP, NADH, NADPH, cellular ROS, and LPO can be higher in glycophytes (such as Brassica juncea; salt-sensitive) when compared with halophytes (such as Sesuvium portulacastrum; salt-tolerant; Srivastava et al.). Redox active compounds, AsA and GSH can play a significant role in the protection of plants against a number of abiotic stresses including temperature (low/chilling and high) and drought (Anjum et al.; Awasthi et al.; Das and Roychoudhury; Lukatkin et al.). Notably, the cellular level of AsA and GSH can differ in Vigna radiata during its ontogeny under drought exposure (Anjum et al.). In addition, sulfur nutrition was revealed as a potential strategy for the management of improved cellular pools of these redox active compounds. The role of polyamines and brassinosteroids for the maintenance of the cellular redox homeostasis in plants exposed to major abiotic stresses was critically discussed by Saha et al. and Vardhini and Anjum, respectively. Montero-Palmero et al. showed that plant endogenous factors like ethylene can modulate the early oxidative stress induced by mercury $(\mathrm{Hg})$. Monoterpenoid indole alkaloids and phenols can be used as a defense tool against stress factors and can also benefit plants depleted in GSH (Vera-Reyes et al.). Dehydrogenases involved in the regeneration of NADPH such as glucose-6-phosphate dehydrogenase (G6PDH), 6phosphogluconate dehydrogenase (6PGDH), NADP-malic enzyme (NADP-ME), and NADP-isocitrate dehydrogenase (NADP-ICDH) can support the protection of plants against nitro-oxidative stress induced by adverse environmental conditions (Corpas and Barroso). Information is scanty in the literature on the mechanisms involved in the control of each mitochondrial enzyme at the post-translational level (Millar et al., 2011; Nunes-Nesi et al., 2013). To this end, Yoshida and Hisabori evidenced that oxidation can inactivate mitochondrial isocitrate dehydrogenase; whereas, the later can be reactivated by thioredoxin-dependent reduction in Arabidopsis.

Insights into the role of redox active compounds AsA and GSH, proteinaceous thiol members such as thioredoxins, peroxiredoxins, and glutaredoxins, electron carriers and energy metabolism mediators phosphorylated (NADP) and nonphosphorylated $(\mathrm{NAD}+)$ coenzyme in the ROS-metabolism and the maintenance of redox homeostasis in abiotic stressedplants were also critically discussed (Kapoor et al.). Notably, the enzymes of the AsA-GSH pathway, a key part of the network of reactions involving enzymes and metabolites with redox properties can have various subcellular isoforms, differ from each other (with respect to their spatial and temporal expression), and can also be differentially regulated by stress types (Anjum et al., 2010, 2012a,b; Gill and Tuteja, 2010). The knowledge gap available on the major mechanisms underlying the regulation of major isoforms of the AsA-GSH pathway enzymes was provided by Pandey et al. These authors provided major insights into the gene families of the AsA-GSH pathway, and also described their roles in the management of cellular redox homeostasis in plants exposed to abiotic and biotic stress conditions. Carvalho et al. elaborated the information available on the main mechanisms underlying plant tolerance to stresses (abiotic and biotic) via phenolic compounds (such as simple flavonoids like anthocyanins). Furthermore, it has recently been demonstrated that condensed proanthocyanidins (tannins) are solubilized into the vacuole or linked to cell wall polysaccharides and largely control the nutraceutical properties of the grape berry and its derivatives such as wine (Tenore et al., 2011; De Nisco et al., 2013).

An improved cellular redox homeostasis and plant-tolerance to environmental challenges are also achieved by employing several seed/plant-priming strategies (Tanou et al.; Bhanuprakash and Yogeesha, 2016). Notably, extreme abiotic and biotic stresses can severely impact or kill the organisms; whereas, low stress levels can exhibit priming effects and benefit stressed-plants (Hadacek and Bachmann). In an attempt to understand this idiosyncratic phenomenon, Hadacek and Bachmann explored the phenomenon of life from a more chemical perspective, elaborated insights into chemical structure diversity and recapitulated the basic reaction chemistry of low-molecularweight metabolites (LMWMs). Additionally, contributions of LMWMs to a homeodynamic systems chemistry of living organisms were also dissected. Non-metabolized and/or elevated levels of ROS (and its reaction products) are the major violators of cellular redox homeostasis in stressed plants. However, the science of cellular redox homeostasis is lagging behind due to a major problem related with the quantification ROS and also with the identification of their short lifetime. Thus, the technique of electron paramagnetic resonance (EPR) spectroscopy could be a panacea to the said issue and can allow disentangling the origin of specific ROS and transient alterations in their cellular levels (Steffen-Heins and Steffens).

Herein, research reports discussing "cellular redox homeostasis in plants" in context with stresses caused by climatic changes (such as drought and salinity) and toxic 
chemicals (such as Hg) were least explored. Also, report is scanty on the "cellular redox homeostasis in plants" vs. biotic factors. However, contributions gathered herein concluded that the "cellular redox homeostasis in plants" is central to the plant stress defense, and the future investigations in this area can help in dissecting more insights into plant responses to environmental stresses. Recent advances in the subject were nicely presented and elaborated, and also identified and listed important open questions and challenges in the article types. Further, this research topic advocates to intensify and integrate biochemical, physiological and molecular-genetic studies on various aspects of the "cellular redox homeostasis in plants." Contributions included in this e-book can be useful for budding scientists working on the subject, and can also encourage further dialog, research and development on the "cellular redox homeostasis in plants."

\section{AUTHOR CONTRIBUTIONS}

NAA prepared the first draft of this "Editorial." All authors listed, have made substantial, direct and intellectual contribution to the work, and approved it for publication.

\section{REFERENCES}

Adem, G. D., Roy, S. J., Zhou, M., Bowman, J. P., and Shabala, S. (2014). Evaluating contribution of ionic, osmotic and oxidative stress components towards salinity tolerance in barley. BMC Plant Biol. 14:113. doi: 10.1186/1471-2229-14-113

Anjum, N. A., Ahmad, I., Mohmood, I., Pacheco, M., Duarte, A. C., et al. (2012a). Modulation of glutathione and its related enzymes in plants' responses to toxic metals and metalloids - a review. Environ. Exp. Bot. 75, 307-324. doi: 10.1016/j.envexpbot.2011.07.002

Anjum, N. A., Umar, S., and Ahmad, A. (2012b). Oxidative Stress in Plants: Causes, Consequences and Tolerance. New Delhi: IK International Publishing House.

Anjum, N. A., Umar, S., and Chan, M. T. (2010). Ascorbate-Glutathione Pathway and Stress Tolerance in Plants. Dordrecht: Springer.

Baxter, A., Mittler, R., and Suzuki, N. (2014). ROS as key players in plant stress signalling. J. Exp. Bot. 65, 1229-1240. doi: 10.1093/jxb/ert375

Bhanuprakash, K., and Yogeesha, H. S. (2016). "Seed priming for abiotic stress tolerance: an overview," in Abiotic Stress Physiology of Horticultural Crops, eds N. K. Srinivasa Rao, K. S. Shivashankara, and R. H. Laxman (New Delhi: Springer India), 103-117.

De Nisco, M., Manfra, M., Bolognese, A., Sofo, A., Scopa, A., Tenore, G. C., et al. (2013). Nutraceutical properties and polyphenolic profile of berry skin and wine of Vitis vinifera L. (cv. Aglianico). Food Chem. 140, 623-629. doi: 10.1016/j.foodchem.2012.10.123

Dietz, K. J. (2003). Redox control, redox signaling, and redox homeostasis in plant cells. Intl. Rev. Cytol. 228, 141-193. doi: 10.1016/S0074-7696(03)28004-9

Foyer, C. H., and Noctor, G. (2009). Redox regulation in photosynthetic organisms: signaling, acclimation, and practical implications. Antiox. Redox Signal 11, 861-905. doi: 10.1089/ars.2008.2177

Foyer, C. H., and Noctor, G. (2011). Ascorbate and glutathione: the heart of the redox hub. Plant Physiol. 155, 2-18. doi: 10.1104/pp.110.167569

Foyer, C. H., and Noctor, G. (2012). Managing the cellular redox hub in photosynthetic organisms. Plant Cell Environ. 35, 199-201. doi: 10.1111/j.13653040.2011.02453.x

Gill, S. S., and Tuteja, N. (2010). Reactive oxygen species and antioxidant machinery in abiotic stress tolerance in crop plants. Plant Physiol. Biochem. 48, 909-930. doi: 10.1016/j.plaphy.2010.08.016

Giordano, M. (2013). Homeostasis: an underestimated focal point of ecology and evolution. Plant Sci. 211, 92-101. doi: 10.1016/j.plantsci.2013.07.008

\section{FUNDING}

NAA gratefully acknowledges the partial financial supports received from FCT (Government of Portugal) through contracts (SFRH/BPD/64690/2009 and SFRH/BPD/84671/2012), the Aveiro University Research Institute/CESAM (UID/AMB /50017/2013), and "COMPETE" through Project n. ${ }^{\circ}$ FCOMP-01-0124-FEDER-02800 (FCT PTDC/AGR-PRO/40 91/2012).

\section{ACKNOWLEDGEMENTS}

We are thankful to contributors for their significant contributions, and also to the reviewers who provided their timely evaluation of the manuscripts. We are also grateful to Prof. Donat Häder for providing us an opportunity to work on this exciting research topic. Timely help rendered by the Front. Environ. Sci. editorial office team is also appreciable that made our efforts successful in bringing out the present treatise on "Redox Homeostasis Managers in Plants under Environmental Stresses."

Millar, A. H., Whelan, J., Soole, K. L., and Day, D. A. (2011). Organization and regulation of mitochondrial respiration in plants. Annu. Rev. Plant Biol. 62, 79-104. doi: 10.1146/annurev-arplant-042110-103857

Nunes-Nesi, A., Araújo, W. L., Obata, T., and Fernie, A. R. (2013). Regulation of the mitochondrial tricarboxylic acid cycle. Curr. Opin. Plant Biol. 16, 335-343. doi: 10.1016/j.pbi.2013.01.004

Oelze, M. L., Kandlbinder, A., and Dietz, K. J. (2008). Redox regulation and overreduction control in the photosynthesizing cell: complexity in redox regulatory networks. Biochim. Biophys. Acta 1780, 1261-1272. doi: 10.1016/j.bbagen.2008.03.015

Osakabe, Y., Osakabe, K., and Shinozaki, K. (2013). "Plant environmental stress responses for survival and biomass enhancement," in Climate Change and Plant Abiotic Stress Tolerance, eds N. Tuteja, and S. S. Gill (Weinheim, Germany: Wiley-VCH Verlag GmbH \& Co. KGaA), 79-108.

Redondo-Gómez, S. (2013). "Abiotic and biotic stress tolerance in plants," in Molecular Stress Physiology of Plants, eds G. R. Rout and A. B. Das (New Delhi: Springer India), 1-20.

Schafer, F. Q., and Buettner, G. R. (2001). Redox environment of the cell as viewed through the redox state of the glutathione disulfide/glutathione couple. Free Radic. Biol. Med. 30, 1191-1212. doi: 10.1016/S0891-5849(01)00480-4

Suzuki, N., Koussevitzky, S., Mittler, R. O. N., and Miller, G. A. D. (2012). ROS and redox signalling in the response of plants to abiotic stress. Plant Cell Environ. 35, 259-270. doi: 10.1111/j.1365-3040.2011.02336.x

Tenore, G. C., Troisi, J., Di Fiore, R., Manfra, M., and Novellino, E. (2011). Nutraceutical value and toxicological profile of selected red wines from Morocco. Food Chem. 129, 792-798. doi: 10.1016/j.foodchem.2011. 05.022

Conflict of Interest Statement: The authors declare that the research was conducted in the absence of any commercial or financial relationships that could be construed as a potential conflict of interest.

Copyright (C) 2016 Anjum, Khan, Sofo, Baier and Kizek. This is an open-access article distributed under the terms of the Creative Commons Attribution License (CC BY). The use, distribution or reproduction in other forums is permitted, provided the original author(s) or licensor are credited and that the original publication in this journal is cited, in accordance with accepted academic practice. No use, distribution or reproduction is permitted which does not comply with these terms. 